\title{
Pair Correlations of an Expanding Superfluid Fermi Gas
}

\author{
C. Lobo, ${ }^{*}$ I. Carusotto, S. Giorgini, A. Recati, and S. Stringari \\ Dipartimento di Fisica, Università di Trento and CNR-INFM BEC Center, I-38050 Povo, Trento, Italy
}

(Received 11 April 2006; published 8 September 2006)

\begin{abstract}
The pair correlation function of an expanding gas is investigated with an emphasis on the BEC-BCS crossover of a superfluid Fermi gas at zero temperature. At unitarity quantum Monte Carlo simulations reveal the occurrence of a sizable bunching effect due to interactions in the spin up-down channel which, at short distances, is larger than that exhibited by thermal bosons in the Hanbury-Brown-Twiss effect. We propose a local equilibrium ansatz for the pair correlation function which we predict will remain isotropic during the expansion even if the trapping potential is anisotropic, in contrast with the behavior of the density. The isotropy of the pair correlation function is an experimentally accessible signature, which makes a clear distinction with respect to the case of noninteracting gases and can be understood as a consequence of the violation of scaling.
\end{abstract}

DOI: 10.1103/PhysRevLett.97.100405

PACS numbers: 03.75.Ss, 03.75.Be, 03.75.Hh

Recent experimental studies of two-body correlations in an expanding atomic cloud are opening new perspectives in the study of quantum-statistical effects in ultracold gases. These include the measurement of the HanburyBrown-Twiss effect [1] in a dilute Bose gas, both concerning spatial and temporal correlations [2,3], measurements in the Mott insulating phase of ultracold gases in an optical lattice [4], as well as the study of atomic pair correlations in a Fermi gas after dissociation of molecules near a Feshbach resonance [5]. In some cases the measured quantities are related to the real space properties (i.e., to the pair correlation function), whereas in other cases they refer to correlations in momentum space. In all cases, measurements are done after a period of free expansion since the gas in situ does not provide sufficient optical resolution.

The pair correlation function is defined as

$$
g_{\sigma \sigma^{\prime}}^{(2)}\left(\mathbf{r}_{1}, \mathbf{r}_{2}\right)=\frac{n^{(2)}\left(\mathbf{r}_{1}, \sigma ; \mathbf{r}_{2}, \sigma^{\prime}\right)}{n\left(\mathbf{r}_{1}, \sigma\right) n\left(\mathbf{r}_{2}, \sigma^{\prime}\right)},
$$

where

$$
n^{(2)}\left(\mathbf{r}_{1}, \sigma ; \mathbf{r}_{2}, \sigma^{\prime}\right)=\left\langle\psi_{\sigma}^{\dagger}\left(\mathbf{r}_{1}\right) \psi_{\sigma^{\prime}}^{\dagger}\left(\mathbf{r}_{2}\right) \psi_{\sigma^{\prime}}\left(\mathbf{r}_{2}\right) \psi_{\sigma}\left(\mathbf{r}_{1}\right)\right\rangle
$$

is the second order correlation function and $n(\mathbf{r}, \sigma)$ is the density of the $\sigma$ spin component. The spin indices $\sigma, \sigma^{\prime}$ are used if there is more than one species of atoms present. The pair correlation function is a key quantity in many-body physics, being sensitive to both statistical and interaction effects. The statistical effects appear, for example, in noninteracting gases of identical bosons above the critical temperature, where they are responsible for a characteristic bunching effect which has been recently the object of experimental measurements [2].

In this Letter we discuss the case of a Fermi gas, with equal population of two spin states, close to a Feshbach resonance where the value of the scattering length $a$ can be tuned by varying the external magnetic field. The opposite spin $g_{\uparrow \downarrow}^{(2)}$ correlation function is strongly affected by inter- actions and for a homogeneous system has been calculated using fixed-node diffusion Monte Carlo simulations [6]. The result at $T=0$ is shown in Fig. 1 where $g_{\uparrow \downarrow}^{(2)}(s)$ is plotted as a function of the dimensionless variable $k_{F} s$ for various configurations. In the inset we plot the integrated quantity $N_{\uparrow \downarrow}(s)=n \int_{0}^{s} 4 \pi r^{2} d r g_{\uparrow \downarrow}^{(2)}(r)$ giving the average number of atoms of opposite spin within a sphere of radius $s$ around a given atom. Here $n=k_{F}^{3} /\left(6 \pi^{2}\right)$ is the single spin density and $k_{F}$ is the Fermi momentum. On the BEC side of the resonance $\left(k_{F} a=0.25\right.$, dashed line) there is a pronounced bunching effect at short distances due to the presence of diatomic molecules and the pair correlation function rapidly approaches the uncorrelated value

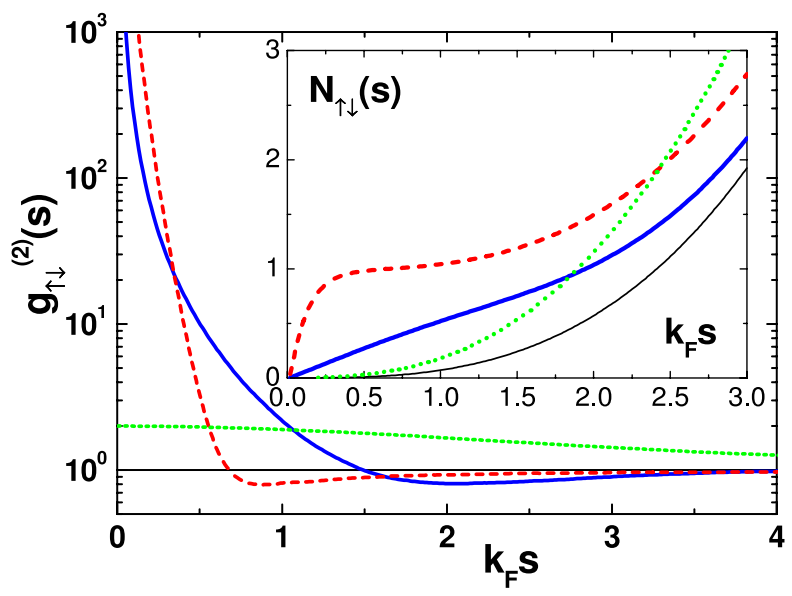

FIG. 1 (color online). Spin up-down pair correlation function $g_{\uparrow \downarrow}^{(2)}(s)$ of a homogeneous system. Dashed line (red online): Fermi gas in the deep BEC regime $\left(1 / k_{F} a=4\right)$; solid line (blue online): Fermi gas at unitarity $\left(1 / k_{F} a=0\right)$; dotted line (green online): ideal Bose gas with density $n=k_{F}^{3} /\left(6 \pi^{2}\right)$ at the Bose-Einstein critical temperature; thin solid line (black online): uncorrelated gas with $g^{(2)}(s)=1$. Inset: integrated pair correlation function $N_{\uparrow \downarrow}(s)$ for the same configurations. 
$g_{\uparrow \downarrow}^{(2)}(s)=1$ (thin solid line) at distances of the order of the scattering length. As a consequence, the integrated probability $N_{\uparrow \downarrow}(s)$ reaches 1 at $k_{F} s \sim 0.25$ in contrast to the uncorrelated gas where the same value is reached much later, at $k_{F} s=2.4$. In the unitary limit $\left(1 / k_{F} a=0\right.$, solid line) $g_{\uparrow \downarrow}^{(2)}$ is a universal function of $k_{F} s$, the inverse of $k_{F}$ providing the only length scale of the problem. In this case the bunching effect is reduced as compared with the BEC case, but is still important (for example, at $k_{F} s=1$ the value of $N_{\uparrow \downarrow}(s)$ is 0.5 whereas the noninteracting value is negligible). For comparison we plot the pair correlation function for a gas of noninteracting bosons with density $n=k_{F}^{3} /\left(6 \pi^{2}\right)$ at the Bose-Einstein critical temperature $T_{c}$ (dotted line). At short distances $g^{(2)}(s)=2$ and the corresponding integrated probability $N(s)$, while larger than that of the uncorrelated gas, is still quite small compared to the unitary case [7]. The sizable effect exhibited by the $g_{\uparrow \downarrow}^{(2)}(s)$ correlation function in interacting Fermi gases is due to the presence of correlations resulting in a $1 / s^{2}$ behavior as $s \rightarrow$ 0 [8] which characterizes not only the deep BEC limit, but the whole crossover [9]. The short range behavior of $g_{\uparrow \downarrow}^{(2)}$ has recently been the subject of experimental studies using spectroscopic techniques [10]. At unitarity, the $s \rightarrow 0$ limit of $s^{2} g_{\uparrow \downarrow}^{(2)}(s)$ is fixed by many-body effects and the quantum Monte Carlo simulation yields the value $\left(k_{F} s\right)^{2} g_{\uparrow \downarrow}^{(2)}(s) \rightarrow$ 2.7. At $1 / k_{F} a=-1$, on the BCS side of the resonance, one finds instead $\left(k_{F} s\right)^{2} g_{\uparrow \downarrow}^{(2)}(s) \rightarrow 0.36$. In the BEC limit the short range behavior of $g_{\uparrow \downarrow}^{(2)}$ is determined by the molecular wave function and one gets the result $\left(k_{F} s\right)^{2} g_{\uparrow \downarrow}^{(2)}(s) \rightarrow 3 \pi /\left(k_{F} a\right)$. Interestingly, the BethePeierls boundary conditions on the many-body wave function [see Eq. (9) below] imply that $\left(s^{2} g\right)^{\prime} / s^{2} g=-2 / a$, which is a nontrivial constraint on $g$.

As already mentioned, it is difficult to directly measure the pair correlation function in real space while the atomic gas is confined in the trap since the cloud is too small to optically resolve features at interatomic distances [11]. The gas needs to be released, undergoing an expansion, before any measurement can be made. If the scattering length is kept constant during the expansion, the calculation of the time dependent evolution of the pair correlation function is strongly affected by interactions and represents a challenging theoretical problem for which few exact solutions are known. One of them is the unitary Fermi gas released from an isotropic harmonic potential $[12,13]$. In the more general case (including anisotropic traps or systems which are not at unitarity) the behavior of the expansion can be nevertheless predicted if the system is in the hydrodynamic (HD) regime, corresponding to a local equilibrium condition. In this case one can use the ansatz

$$
g^{(2)}\left(\mathbf{r}_{1}, \mathbf{r}_{2}, t\right)=g_{\text {hom }}^{(2)}(\mathbf{s}, n(\mathbf{r}, t)),
$$

for the pair correlation function, based on a local density approximation. Here $\mathbf{r}=\left(\mathbf{r}_{1}+\mathbf{r}_{2}\right) / 2$ and $\mathbf{s}=\mathbf{r}_{1}-\mathbf{r}_{2}$ are, respectively, the center of mass and relative coordinates and $g_{\text {hom }}^{(2)}$ is the pair correlation function for a homogeneous gas with density $n(\mathbf{r}, t)$. From an experimental point of view the ansatz (3) relates the locally measured density $n(\mathbf{r}, t)$ to the locally measured $g^{(2)}$. From a theoretical perspective it gives us the value of $g^{(2)}$ during the expansion if we know the equilibrium form of $g_{\text {hom }}^{(2)}(s)$ (from, e.g., quantum Monte Carlo calculations) and the time evolution of the local density $n(\mathbf{r}, t)$. In particular, at unitarity, where $g_{\text {hom }}^{(2)}$ depends only on the combination $k_{F} s$, the expansion acts like a microscope, the value of $k_{F} \propto$ $n^{1 / 3}$ being reduced as a function of time.

The dynamics of the density $n(\mathbf{r}, t)$ can be analyzed employing the HD theory. This theory has been quite successful in predicting the behavior of $n(\mathbf{r}, t)$ in several important configurations. For example, in the case of a dilute Bose gas, the hydrodynamic predictions for the time evolution of the density agree in an excellent way with the exact solutions of the Gross-Pitaevskii equation and with experiments [see, for example, Ref. [14]]. They have been used to study, among others, the collective oscillations [15] and the expansion after release from the trap [16-18]. If the trapping has the harmonic form $V(\mathbf{r})=$ $\sum_{i=x, y, z} m \omega_{i}^{2} r_{i}^{2} / 2$ and the chemical potential follows a power law dependence on the density, $\mu(n) \propto n^{\gamma}$ the HD predictions for the expanding density profile can be written in the scaled form

$$
n(\mathbf{r}, t)=\frac{1}{b_{x} b_{y} b_{z}} n_{0}\left(x / b_{x}, y / b_{y}, z / b_{z}\right),
$$

with the scaling coefficients $b_{i}$ obeying the system $[16,17]$

$$
\ddot{b}_{i}-\frac{\omega_{i}^{2}}{b_{i}\left(b_{x} b_{y} b_{z}\right)^{\gamma}}=0,
$$

of nonlinear coupled equations with the initial conditions $b_{i}(t=0)=1$ and $n_{0}(\mathbf{r}) \equiv n(\mathbf{r}, t=0)$. The polytropic dependence of the chemical potential applies both to the case of a dilute Bose gas $(\gamma=1)$ and to the unitary limit of a Fermi gas $(\gamma=2 / 3)$. When released from an anisotropic trap, the aspect ratio of the expanding density profile exhibits an inversion from the cigar to the disc geometry (and vice versa) as a consequence of the HD forces which are more active along the directions where the gradient of the density is larger. Therefore, the density profile at long times will be anisotropic. On the other hand, we see from Eq. (3) that the pair correlation function will keep its isotropy as a function of $\mathbf{s}$. We should also point out that the gas does not need to be superfluid in order to be in the HD regime and to exhibit isotropy of $g^{(2)}$ during expansion [19]).

The ansatz (3), which assumes local equilibrium for the description of the expansion, requires more stringent conditions compared to the usual local density approximation applied to stationary configurations. In the latter case, the 
only relevant condition is that the typical length scale, where $g^{(2)}$ approaches the uncorrelated value $g^{(2)}=1$, be much smaller than the size of the cloud. The use of Eq. (3), instead, also requires that adiabaticity be ensured during the expansion. In the superfluid regime, HD theory provides a justification for the local equilibrium approximation. We might, however, wonder whether superfluidity is preserved during the expansion. In a Fermi gas at $T=0$, the disappearance of superfluidity could take place through pair breaking mechanisms. Since a large number of quantized vortices are observed after expansion, recent experiments [20] suggest that the system remains superfluid during the expansion on the BEC side of the crossover. The robustness of superfluidity is also suggested by the fact that the gap is much larger than the typical oscillator frequency (whose inverse is the time scale of the expansion) and that, as a consequence, pairs cannot easily break during the expansion. This argument holds also at unitarity where the gap is of the order of the Fermi energy. On the other hand, superfluidity is more fragile on the BCS side because the gap becomes exponentially small during the expansion. For a measurement of the pairing gap in ultracold Fermi gases along the BEC-BCS crossover, see Ref. [21].

The experimental measurement of the pair correlation function and the verification of Eq. (3) requires that certain conditions be met. In particular, the expansion time should be long enough for features at the interatomic level to be resolved. By choosing, for example, a trapped gas with central density equal to $n \simeq 10^{13} \mathrm{~cm}^{-3}$ (corresponding to an inverse Fermi momentum equal to $k_{F}^{-1} \simeq 100 \mathrm{~nm}$ ) and isotropic trapping, we find that, after a time equal to $\omega t \simeq$ 20 , the density is reduced to a value $\sim 10^{9} \mathrm{~cm}^{-3}$ corresponding to $k_{F}^{-1} \sim 3 \mu \mathrm{m}$. This value is compatible with the present limits of optical resolution [22]. At the same time, if we choose a large value of the scattering length the system will keep the condition of unitarity $k_{F} a \gg 1$ also at the observation time.

The HD description of the expansion points out the occurrence of important qualitative differences in the expansion of interacting and noninteracting gases. In fact, one of the easiest ways of seeing the effects of interactions in experiments is to measure the isotropy or anisotropy in the spatial profiles of observable quantities.

A summary of our predictions is given in Table I. Contrary to what happens in the HD regime, when released from an anisotropic trap, the density of a noninteracting gas (e.g., fermions or uncondensed thermal bosons) be-

TABLE I. Role of anisotropy in the expansion of the density and of the pair correlation function of a trapped gas.

\begin{tabular}{lcr}
\hline \hline Behavior after expansion & $n(\mathbf{r}, t)$ & $g^{(2)}(\mathbf{s}, t)$ \\
\hline $\begin{array}{l}\text { Ideal gas } \\
\text { (bosons } T>T_{c} \text { or fermions) }\end{array}$ & isotropic & anisotropic \\
Hydrodynamic regime & anisotropic & isotropic \\
\hline \hline
\end{tabular}

comes isotropic at long times during the expansion, reflecting the isotropy of the initial momentum distribution [23]. The pair correlation function also exhibits drastic differences. In fact, in a noninteracting gas the dependence of $g^{(2)}$ on the relative coordinate $\mathbf{s}$ will undergo an anisotropic expansion. For example, for noninteracting bosonic or fermionic gases trapped in a harmonic potential at temperatures much higher than that of quantum degeneracy, the pair correlation function can be shown to exhibit the following behavior [19]

$$
g^{(2)}(\mathbf{s}, t)=1 \pm \exp \left(-2 \pi \sum_{i=x, y, z} \frac{s_{i}^{2}}{\lambda_{T}^{2}\left(\omega_{i}^{2} t^{2}+1\right)}\right),
$$

where $\lambda_{T}=\sqrt{2 \pi \hbar^{2} / m k_{B} T}$ is the thermal de Broglie wavelength. Here the plus sign is for bosons, revealing a bunching effect (as discussed previously), while the minus sign is for fermions which exhibit antibunching as a consequence of the Pauli principle. For large times, the typical value of the decay length of these statistical correlations depends on direction and scales as $\lambda_{T} \omega_{i} t=\sqrt{4 \pi} \hbar t /\left(m R_{i}\right)$, a value that has recently been used to analyze the data in the experiment of [2] with thermal bosons. Here $R_{i}=\sqrt{2 k_{B} T / m \omega_{i}^{2}}$ is the in situ radius of the thermal cloud in the $i$ th direction.

Let us finally discuss the problem of the expansion from the general point of view of scaling, characterized by a coordinate transformation of the type:

$$
x \rightarrow x / b_{x}(t), \quad y \rightarrow y / b_{y}(t), \quad z \rightarrow z / b_{z}(t) .
$$

An exact scaling solution is known to be obeyed by the many-body wave function of a unitary Fermi gas released from an isotropic harmonic potential [12]. In this case all correlation functions exhibit the same scaling behavior and one finds $n(\mathbf{r}, t)=n_{0}(\mathbf{r} / b) / b^{3}$ and $n^{(2)}\left(\mathbf{r}_{1}, \mathbf{r}_{2}, t\right)=$ $n^{(2)}\left(\mathbf{r}_{1} / b, \mathbf{r}_{2} / b, 0\right) / b^{6}$, with $b=\sqrt{\omega^{2} t^{2}+1}$. The HD picture reproduces this behavior; in fact, Eqs. (4) and (5), with $\omega_{i} \equiv \omega, b_{i}(t) \equiv b(t)$, and $\gamma=2 / 3$, yield the same time dependence for the density profile. Agreement is also found for the time dependence of the pair correlation function. In fact at unitarity the correlation function evaluated for a homogeneous medium depends only on the combination $k_{F} s \propto n^{1 / 3} s$ so that Eq. (3) can be written in the scaled form

$$
g^{(2)}\left(\mathbf{r}_{1}, \mathbf{r}_{2}, t\right)=g_{\text {hom }}^{(2)}\left[n_{0}^{1 / 3}(\mathbf{r} / b) s / b\right],
$$

which, for isotropic harmonic trapping, agrees with the scaling result $g^{(2)}\left(\mathbf{r}_{1}, \mathbf{r}_{2}, t\right)=g^{(2)}\left(\mathbf{r}_{1} / b, \mathbf{r}_{2} / b, 0\right)$. Naturally the agreement holds only for values of $s$ much smaller than the size of the system where the local density approximation can be properly applied.

The validity of scaling to describe the expansion of the pair correlation function is, however, not general. At distances much shorter than the interparticle separation, but much larger than the effective range of interatomic interactions, the wave function is determined up to a propor- 
tionality constant by the Bethe-Peierls boundary condition: for any value of the scattering length $a$ and for all pairs of atoms $i j$, the many-body wave function $\Psi$ obeys the condition

$$
\Psi\left(\left|\mathbf{r}_{i}-\mathbf{r}_{j}\right| \rightarrow 0\right) \propto \frac{1}{\left|\mathbf{r}_{i}-\mathbf{r}_{j}\right|}-\frac{1}{a},
$$

which is in general incompatible with scaling. This boundary condition has two characteristic features: (i) it is isotropic and (ii) it introduces a length scale $a$. From (i) we can now better understand the behavior of $g^{(2)}$ summarized in the table. It implies that $g^{(2)}$ must remain isotropic (at least for small $\left|\mathbf{r}_{1}-\mathbf{r}_{2}\right|$ ) for all interacting gases whereas in the noninteracting limit this constraint is removed and $g^{(2)}$ is allowed to exhibit the anisotropic scaling of Eq. (6). On the other hand, (ii) means that a rescaling of the coordinates would change the ratio $a /\left|\mathbf{r}_{i}-\mathbf{r}_{j}\right|$ which is required to be fixed. Such an argument applies to $g^{(2)}$ and to all higher order correlation functions as they all obey the boundary condition (9). However, it does not affect the density $n(\mathbf{r}, t)$ for which scaling laws do exist, e.g., for HD systems. In particular, (ii) leads to a dramatic difference between the BEC and unitary regimes. The latter has perfect scaling of $g_{\uparrow \downarrow}^{(2)}$ if the gas is isotropic, while the molecular gas has not, even though the density $n(\mathbf{r}, t)$ scales during the expansion as in ordinary Bose-Einstein condensates, since it now contains information about the molecular bound state whose size is fixed only by the scattering length $a$.

In conclusion, we investigated the spin up-down pair correlation function of a Fermi gas at $T=0$ in the BEC$\mathrm{BCS}$ crossover. Under the condition that local equilibrium is ensured during the expansion, we addressed the nontrivial problem of the time dependence of the correlation function after release of the gas from the confining potential. In contrast to the behavior of noninteracting gases, the pair correlation function violates the scaling solution (7) and is predicted to remain locally isotropic during the expansion even for anisotropic trapping. At unitarity, the absence of other relevant length scales beside $k_{F}^{-1}$ makes the expansion act like a magnification lens as the average distance between particles becomes visible.

We gratefully acknowledge useful discussions with L.P. Pitaevskii and M.W. Zwierlein. We also acknowledge support by the Ministero dell'Istruzione, dell'Università e della Ricerca (MIUR).

*Electronic address: clobo@science.unitn.it

[1] See, e.g., R. Loudon, The Quantum Theory of Light (Clarendon, Oxford, 1973); for a measurement of $g^{(2)}$ in atomic beams: M. Yasuda and F. Shimizu, Phys. Rev. Lett. 77, 3090 (1996).

[2] M. Schellekens et al., Science 310, 648 (2005).
[3] A. Öttl, S. Ritter, M. Köhl, and T. Esslinger, Phys. Rev. Lett. 95, 090404 (2005).

[4] S. Fölling et al., Nature (London) 434, 481 (2005).

[5] M. Greiner, C. A. Regal, J. T. Stewart, and D. S. Jin, Phys. Rev. Lett. 94, 110401 (2005).

[6] G.E. Astrakharchik, J. Boronat, J. Casulleras, and S. Giorgini, Phys. Rev. Lett. 93, 200404 (2004).

[7] Furthermore, an important reduction in the pair correlation function of the Bose gas is produced by the inclusion of interactions. See, for example, M. Holzmann and Y. Castin, Eur. Phys. J. D 7, 425 (1999); M. Naraschewski and R. J. Glauber, Phys. Rev. A 59, 4595 (1999).

[8] Notice that $s \rightarrow 0$ here means $R_{0} \ll s \ll \min \left(a, k_{F}^{-1}\right)$, where $R_{0}$ is the effective range of interatomic forces.

[9] The spin up-up correlation function $g_{\Uparrow}^{(2)}$ is less affected by interactions than $g_{\uparrow \downarrow}^{(2)}$. For example, at unitarity, it almost coincides with the prediction for a noninteracting Fermi gas [6].

[10] M. Greiner et al., Phys. Rev. Lett. 92, 150405 (2004); G. B. Partridge et al., Phys. Rev. Lett. 95, 020404 (2005).

[11] An alternative method would consist of measuring the static structure factor $S(k)$, i.e., the Fourier transform of the correlation function. The method, based on twophoton stimulated Bragg scattering, has been already successfully used to measure $S(k)$ of a dilute Bose gas [see J. Stenger et al., Phys. Rev. Lett. 82, 4569 (1999)]. For a recent discussion of the behavior of $S(k)$ in a Fermi gas near a Feshbach resonance, see R. Combescot, S. Giorgini, and S. Stringari, cond-mat/0512048.

[12] Y. Castin, C.R. Physique 5, 407 (2004).

[13] Another nontrivial case where an exact solution is available is the 1D Tonks gas initially trapped by a harmonic potential. In this case $g^{(2)}$ coincides with the one of the noninteracting Fermi gas and evolves in time with a characteristic antibunching behavior at short distances.

[14] F. Dalfovo, S. Giorgini, L. Pitaevskii, and S. Stringari, Rev. Mod. Phys. 71, 463 (1999).

[15] S. Stringari, Phys. Rev. Lett. 77, 2360 (1996).

[16] Y. Castin and R. Dum, Phys. Rev. Lett. 77, 5315 (1996).

[17] Yu. Kagan, E. L. Surkov, and G. V. Shlyapnikov, Phys. Rev. A 54, R1753 (1996).

[18] C. Menotti, P. Pedri, and S. Stringari, Phys. Rev. Lett. 89, 250402 (2002).

[19] In the collisional regime the expansion is instead governed by the adiabaticity condition [17] and the pair correlation function obeys the isotropic law $g^{(2)}(\mathbf{s}, t)=$ $1 \pm \exp \left[-2 \pi \mathbf{s}^{2} / \alpha^{2 / 3}(t) \lambda_{T}^{2}\right]$, with $\alpha(t)=b_{x}(t) b_{y}(t) b_{z}(t)$ and $b_{i}$ given by the solution of the hydrodynamic Eqs. (5) with $\gamma=2 / 3$. The factor $\alpha^{2 / 3}(t)$ takes into account the reduction of the temperature of the gas during the adiabatic expansion.

[20] M. W. Zwierlein et al., Nature (London) 435, 1047 (2005);

[21] C. Chin et al., Science 305, 1128 (2004).

[22] Notice that the quantity $N_{\uparrow \downarrow}(s)$ plotted in Fig. 1 is related to $g_{\uparrow \downarrow}^{(2)}(s)$ convoluted with a response function which is flat over a sphere of radius $s$ and zero outside.

[23] A noninteracting Bose gas at $T=0$ behaves differently, as the expansion after release is anisotropic. However, in this case, $g^{(2)}(s)=1$ at any time. 